\title{
OPTICAL PROPERTIES OF DILUTED MAGNETIC SEMICONDUCTORS IN COHERENT POTENTIAL APPROXIMATION
}

\author{
HOANG ANH TUAN \\ Max Planck Institute for the Physics of Complex Systems, Nöthnizer Strasse 38, 01187 \\ Dresden, Germany \\ Institute of Physics and Electronics, BO Box 429 Bo Ho, Hanoi 10000, Vietnam
}

\begin{abstract}
We study optical properties of a single band model for $(I I I, M n) V$ diluted magnetic semiconductors (DMS), treating the impurity spins fully quantum mechanically in the coherent potential approximation (CPA). Our CPA results show an interesting dependence of the optical conductivity on Kondo coupling, carrier density, nonmagnetic potential, and temperature. We find that by taking into account the spin-flip effect the calculated conductivity is in resonable agreement with ones obtained by Monte-Carlo simulations and experiments.
\end{abstract}

\section{INTRODUCTION}

The III-V diluted magnetic semiconductors showing ferromagnetism with Curie temperature $T_{c} \approx 170 \mathrm{~K}$ become one of the most promising materials for spintronic applications [1]. However, in spite of much activity, the nature of ferromagnetism is not properly understood and the parameter control of the magnitude of Curie temperature is still an open question. It is well-known that the magnetism and transport properties in DMS are heavily affected by material issues, e.g., disoder from the doped Mn ions, carrier compensation, the coupling mechanism between the carries and local spins, as well as a complicated band structure [2]. Therefore, it is impossible to study these materials without making some approximations. There are variuos theoretical approaches attempting to understand DMS physics. An overview of the theory of ferromagnetic (III,Mn)V semiconductors was recently presented in Ref. [3].

The optical conductivity is among important probe of DMS materials. In order to theoretical study optical properties of these materials we choose impurity band model and the coherent potential approximation method among various models and approaches for (III,Mn)V DMS. Our choice is based on the followings: (i) The model gives consistent explanation for the mechanism of the carrier-induced ferromagnetism in III-V based materials [4,5], and the Curie temperature obtained by using CPA agrees very well with experiments [6-8]; (ii) The study of the single band model using Monte-Carlo (MC) techniques on finite clusters and the dynamical mean field theory (DMFT) shows that they can contribute a comprehensive understanding of DMS [9,10]; and (iii) The choice of the single band model allows us to focus on the essential aspect of the problem, leaving aside the numerical complexity of the multi-band model, which can not be studied with reliable 
numerical techniques.

In our previous work [11], we calculated the optical conductivity in the model where localized spins are approximated as Ising spins. As in DMFT studies [12], we demonstrated that the formation of a spin-polarized impurity band leads to a finite frequency peak in the optical spectrum. In this work, in order to improve the agreement with the experiments, in particular, the experimentally observed temperature dependence of the optical conductivity, we treat the impurity spins fully quantum mechanically using the formalism of the CPA in Refs. [7, 13]. We find that by taking into account the spin-flip effect the calculated conductivity is in reasonable agreement with ones obtained by Monte-Carlo simulations and experiments.

\section{MODEL AND FORMALISM}

We employ the minimal model of $\left(\mathrm{A}_{1-x} \mathrm{Mn}_{x}\right) \mathrm{B}$ type DMS which includes the exchange interaction and nonmagnetic attractive potential. The Hamiltonian reads

$$
H=\sum_{i j \sigma} t_{i j} c_{i \sigma}^{+} c_{j \sigma}+\sum_{i} u_{i}
$$

where $t_{i j}$ is the hopping matrix element between the site $i$ and $j, u_{i}$ depends on the ion species occupying the $i$ site:

$$
u_{i}=\left\{\begin{array}{lr}
E_{A} \sum_{\sigma} c_{i \sigma}^{+} c_{i \sigma}, & i \in A \\
E_{M} \sum_{\sigma} c_{i \sigma}^{+} c_{i \sigma}+J \mathbf{S}_{i} \mathbf{s}_{i}, & i \in M n
\end{array}\right.
$$

Here $c_{i \sigma}^{+}\left(c_{j \sigma}\right)$ is the creation (annihilation) operator for a carrier at site $i$ with spin $\sigma$, $E_{A}\left(E_{M}\right)$ represents a nonmagnetic local potential at an $\mathrm{A}(\mathrm{Mn})$ site. $\mathbf{S}_{i}$ is the magnetic impurity spin at site $i$ and $\mathbf{s}_{i}=c_{i \alpha}^{+}\left(\frac{1}{2} \sigma_{\alpha \beta}\right) c_{i \beta}$ is the spin operator at site $i$ of the itinerant carrier, $J>0$ is the local Kondo coupling. The difference of the nonmagnetic potential on the impurity atom $(\mathrm{Mn})$ from that on the host atom (A), $E_{M}-E_{A}$, acts as an attractive potential in III-V-based DMS. Unlike previous works $[11,12]$ where local spins are approximated as Ising spins, we treat the impurity spin $(S=5 / 2)$ quantum mechanically. For that purpose we follow the procedure described in Refs. $[7,13]$. The t-matrix associated to the multiscattering of the single magnetic impurity at site $i$ embedded in the effective medium is

$$
t_{i}=v_{i}\left(1-G v_{i}\right)^{-1}
$$

where

$$
v_{i}=\left\{\begin{array}{lr}
\sum_{\sigma}\left(E_{A}-\Sigma_{\sigma}\right) c_{i \sigma}^{+} c_{i \sigma}, & i \in A \\
\sum_{\sigma}\left(E_{M}-\Sigma_{\sigma}\right) c_{i \sigma}^{+} c_{i \sigma}+J \mathbf{S}_{i} \mathbf{s}_{i}, & i \in M n
\end{array}\right.
$$

The $2 \times 2$ average Green function matrix $G$ is $\left(\begin{array}{cc}G_{\uparrow} & 0 \\ 0 & G_{\downarrow}\end{array}\right)$, with $G_{\sigma}=\left(\omega I-K_{\sigma}\right)^{-1}$, where $K_{\sigma}=\sum_{\mathbf{k}}\left(\varepsilon_{\mathbf{k}}+\Sigma_{\sigma}\right) c_{\mathbf{k} \sigma}^{+} c_{\mathbf{k} \sigma}, \varepsilon_{\mathbf{k}}$ is the Fourier transform of $t_{i j}$ and $\Sigma_{\sigma}$ is the CPA self-energy. 
The CPA demands that the scattering matrix vanishes on average:

$$
(1-x)\left\langle t_{\sigma \sigma}^{A}\right\rangle_{T}+x\left\langle t_{\sigma \sigma}^{M n}\right\rangle_{T}=0 .
$$

Here the $t$-matrix elements in the Wannier representation $\left\langle m \sigma|t| m \sigma^{\prime}\right\rangle$ are wrriten as $t_{\sigma \sigma^{\prime}}$. The thermal average at temperature $T$ for spin operators for any function $f\left(S^{z}\right)$ is given by $\left\langle f\left(S^{z}\right)\right\rangle_{T}=\sum_{S^{z}=-S}^{S} e^{\lambda S^{z}} f\left(S^{z}\right) / \sum_{S^{z}=-S}^{S} e^{\lambda S^{z}}$, where $\lambda$ is then determined self-consistently from the Weiss molecular field theory. The explicit form CPA equations $(5)(\sigma= \pm 1)$ are given in $[7,13]$.

The Green function for the effective medium is given by $F_{\sigma}(\varepsilon)=<m \sigma\left|G_{\sigma}\right| m \sigma>=$ $\frac{1}{N} \sum_{\mathbf{k}} \frac{1}{\varepsilon-\varepsilon_{\mathbf{k}}-\Sigma_{\sigma}(\varepsilon)}$, where $N$ is the number of lattice sites. By using the unperturbed density of states (DOS) $\rho_{0}(z)=\frac{2}{\pi W^{2}} \sqrt{W^{2}-z^{2}}$, where $W$ is the half bandwidth, we have $F^{(0)}(\varepsilon)=\frac{2}{W^{2}}\left(\varepsilon-\sqrt{\varepsilon^{2}-W^{2}}\right)$ and $F_{\sigma}(\varepsilon)=F^{(0)}\left(\varepsilon-\Sigma_{\sigma}\right)$. Solving for $\Sigma_{\sigma}$, we get

$$
\Sigma_{\sigma}(\varepsilon)=\varepsilon-\frac{W^{2}}{4} F_{\sigma}(\varepsilon)-\frac{1}{F_{\sigma}(\varepsilon)} .
$$

Using (6), the CPA equations (5) can be turned into equations for $F_{\sigma}(\varepsilon)$. The DOS $\rho_{\sigma}(\varepsilon)$ is then obtained by $\rho_{\sigma}(\varepsilon)=-\frac{1}{\pi} \operatorname{Im} F_{\sigma}(\varepsilon)$.

The carrier density of states can be express as

$$
n=\int_{-\infty}^{\infty}\left[\rho_{\uparrow}(\varepsilon)+\rho_{\downarrow}(\varepsilon)\right] f(\varepsilon) d \varepsilon
$$

where $f(\varepsilon)=\left(1+e^{(\varepsilon-\mu) / k_{B} T}\right)^{-1}$ is the Fermi distribution function, $\mu$ is the chemical potential. Given magnetization of the impurity spin $\left\langle S^{z}\right\rangle_{T}=m$, one can calculate $F_{\sigma}(\varepsilon)$ from equations (5), and then the DOS and magnetization of the carriers $\left\langle s_{z}\right\rangle$. So one can establish the relation $\left\langle s_{z}\right\rangle=\left\langle s_{z}\right\rangle(m)$. By using the Weiss molecular field theory, given $\left\langle s_{z}\right\rangle$ each impurity spin feels an effective field $J\left\langle s_{z}\right\rangle$ and thus we have $m=S B_{S}\left(-J\left\langle s_{z}\right\rangle(m) / k_{B} T\right)$, here $B_{S}(x)$ is the conventional Brillouin function. Since there is one-to-one correspondence between $m$ and $\lambda$, we have a set of self-consistent equations for $\mu$ and $m$ for a given set of parameter values $x, n, J, E_{A}, E_{M}$ and $T$. We note that unlike Refs. [5,7] where the local magnetization $m$ is left as an input parameter, it is determined self-consistently in our calculations.

Knowledge of single particle self-energy enables one to derive the optical conductivity of the disordered system in CPA. Within this approximation $[14,15]$ the real part of the conductivity is given by

$$
\sigma_{x x}(\omega)=\frac{e^{2} \pi}{V \hbar} \int \frac{f(\varepsilon-\omega)-f(\varepsilon)}{\omega} \frac{1}{N} \sum_{\mathbf{k} \sigma}\left(\frac{\partial \varepsilon_{\mathbf{k}}}{\partial k_{x}}\right)^{2} A_{\mathbf{k} \sigma}(\varepsilon-\omega) A_{\mathbf{k} \sigma}(\varepsilon) d \varepsilon,
$$




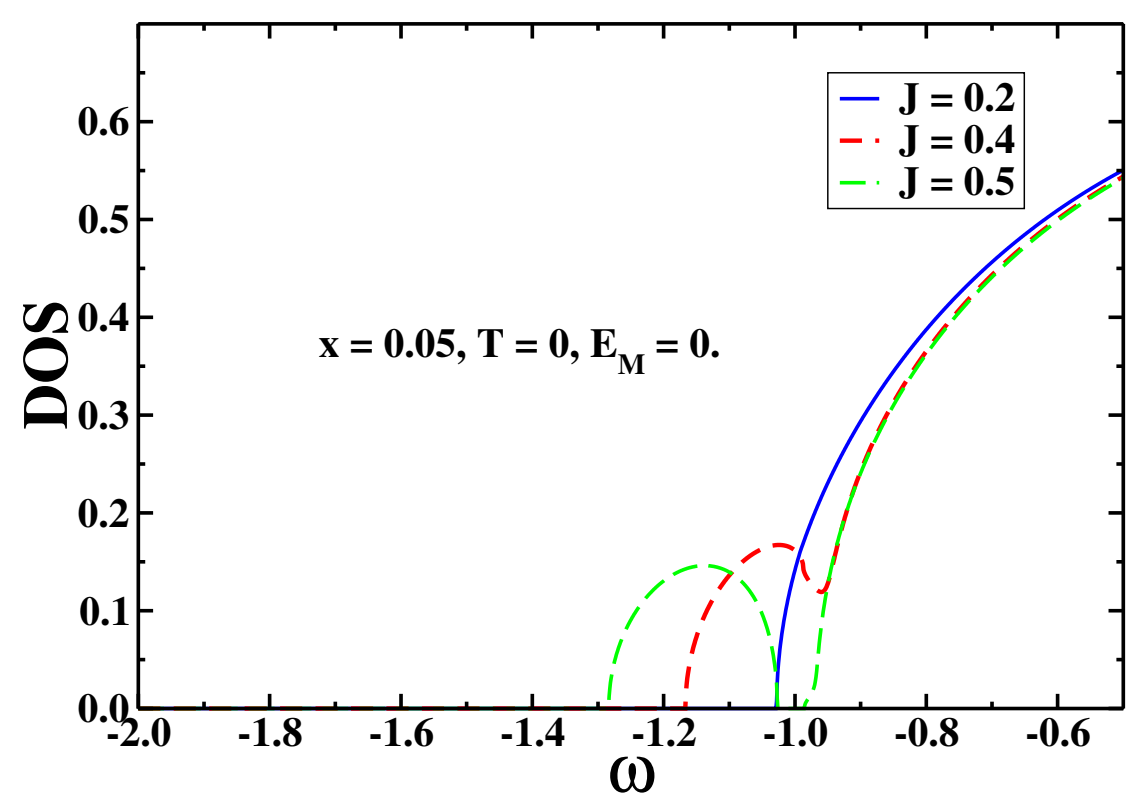

Figure 1: The calculated majority spin DOS for various coupling constants

where $V$ is the cell volume, $A_{\mathbf{k} \sigma}(\varepsilon)=-\frac{1}{\pi} \operatorname{Im}\left(\varepsilon-\varepsilon_{\mathbf{k}}-\Sigma_{\sigma}\right)^{-1}$ is the spectral weight. In the limit of large coordination number and for a simple hypercubic lattice, the $\mathbf{k}$ sum in equation (8) can be further simplified. In semicircular DOS model we obtain

$$
\sigma(\omega)=\frac{\sigma_{x x}}{\sigma_{0}}=\int \frac{f(\varepsilon-\omega)-f(\varepsilon)}{\omega} Y(\varepsilon, \omega) d \varepsilon
$$

with $\sigma_{0}$ being the Mott minimal metallic conductivity, and

$$
\begin{gathered}
Y(\varepsilon, \omega)=\frac{4 \pi W}{3} \sum_{\sigma} \int_{-W}^{W}\left(1-\frac{z^{2}}{W^{2}}\right)^{3 / 2} A_{\sigma}(\varepsilon, z) A_{\sigma}(\varepsilon-\omega, z) d z, \\
A_{\sigma}(\varepsilon, z)=-\frac{1}{\pi} \operatorname{Im} \frac{1}{\varepsilon-z-\Sigma_{\sigma}(\varepsilon)}
\end{gathered}
$$

The static conductivity is found from eqs. (9)-(10) in the limit $\omega \rightarrow 0$.

\section{NUMERICAL RESULTS AND DISCUSSIONS}

Throught this work we take $E_{A}$ as the origin $(=0)$ and $W$ as the unit of energy. In Fig. 1, examples of the low energy part of the majority spin (spin-down) DOS are shown for parameter values $x=0.05$ (we focus on the doping and intermediate couplings associated with the highest $T_{c}$ in $\left.\mathrm{Ga}_{1-x} \mathrm{Mn}_{x} \mathrm{As}\right), T=0, E_{M}=0$ and three values of the Kondo coupling $J$. For weak coupling $(J=0.2 W)$ the impurity band is not formed, for intermediate coupling $(J=0.4 W)$ the impurity band is formed but not well separated from the main band, and for strong coupling $(J=0.5 W)$ we find a separated impurity 


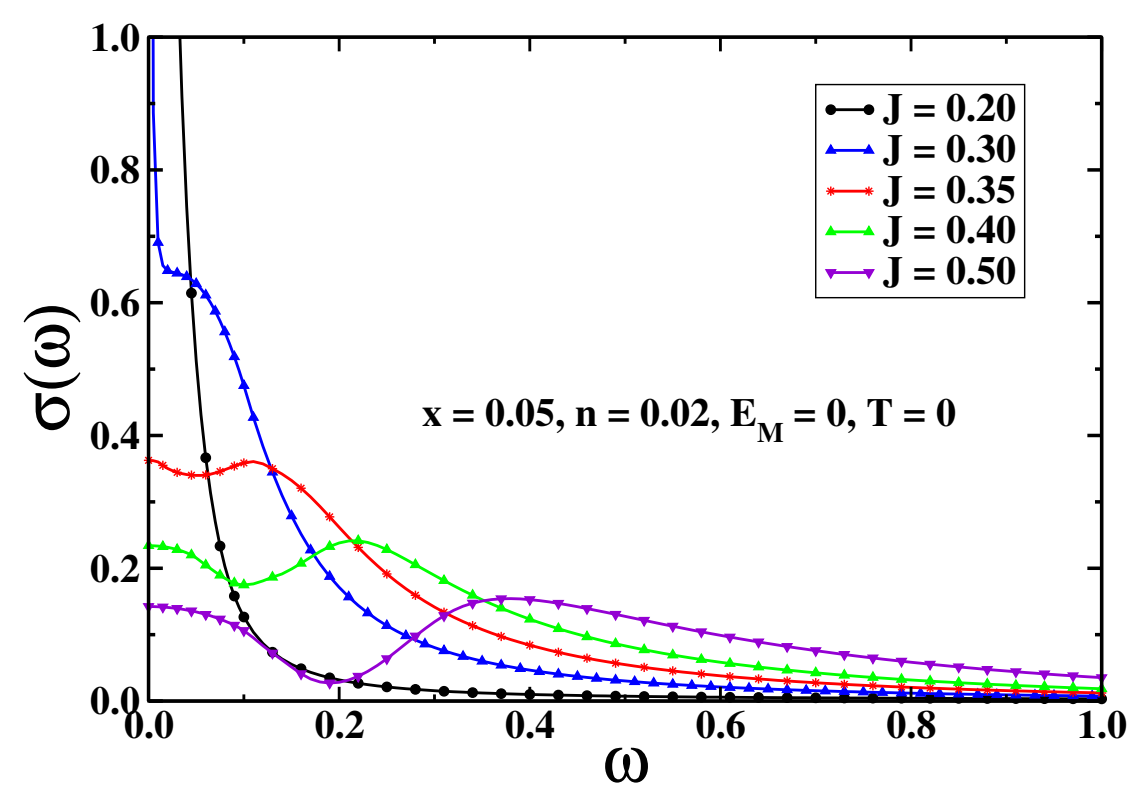

Figure 2: Carrier-spin coupling dependence of the conductivity

band below the main band. As it is well known the carrier density is subtantially smaller than the impurity concentration due to the heavy compensation. Therefore the chemical potential is located in the lower impurity band or the lower band edge, and the key physics issue is whether the Kondo coupling is weak or strong.

Fig. 2 diplays the change of the conductivity with the change coupling $J$ for fixed values of $x, E_{M}, n$ and temperatute $T$. This optical conductivity has two main features: (i) a zero-frequency or Drude peak corresponding to motion within the impurity band or the lower energy band, and (ii) a finite-frequency or non-Drude broad peak corresponding to transitions from the impurity band to the main band. It is seen that non-Drude peak only appears for intermediate and strong couplings. In addition, as the coupling increases, the non-Drude peaks decrease in intensity due to carrier localization. The frequency of this peak, $\omega^{*}$, depens on $J$ and carrier density $n$. For intermediate coupling $(J / W=0.3 \div 0.4)$ and $n=0.02$ we found $0.04 \leq \omega^{*} / W \leq 0.21$. For $\mathrm{Ga}_{1-x} \mathrm{Mn}_{x}$ As the bandwidth $2 W \approx 4 \mathrm{eV}$ $[5,16]$, yieding $0.1 \mathrm{eV} \leq \omega^{*} \leq 0.4 \mathrm{eV}$, in reasonable agreement with the ones obtained by using Monte-Carlo simulation. This result is in semiqualitative agreement with the experiment results for $\mathrm{Ga}_{1-x} \mathrm{Mn}_{x}$ As for $x=0.052$ where a broad peak is at around 0.2 $\mathrm{eV}[17,18]$. The agreement between CPA and MC results, as it was noted in Ref. [19], is due to the fact that at weak and intermediate couplings, the carriers can be sufficiently delocalized that a smearing effect may occur and considering the disorder only in average appears to be sufficient. Moreover, since the actual value of Kondo coupling and the carrier density are not known experimentally with precision, it is difficult to quantitavely compare the calculated results with experiments.

In Fig. 3 we present the evolution of the conductivity with temperature. Here we 


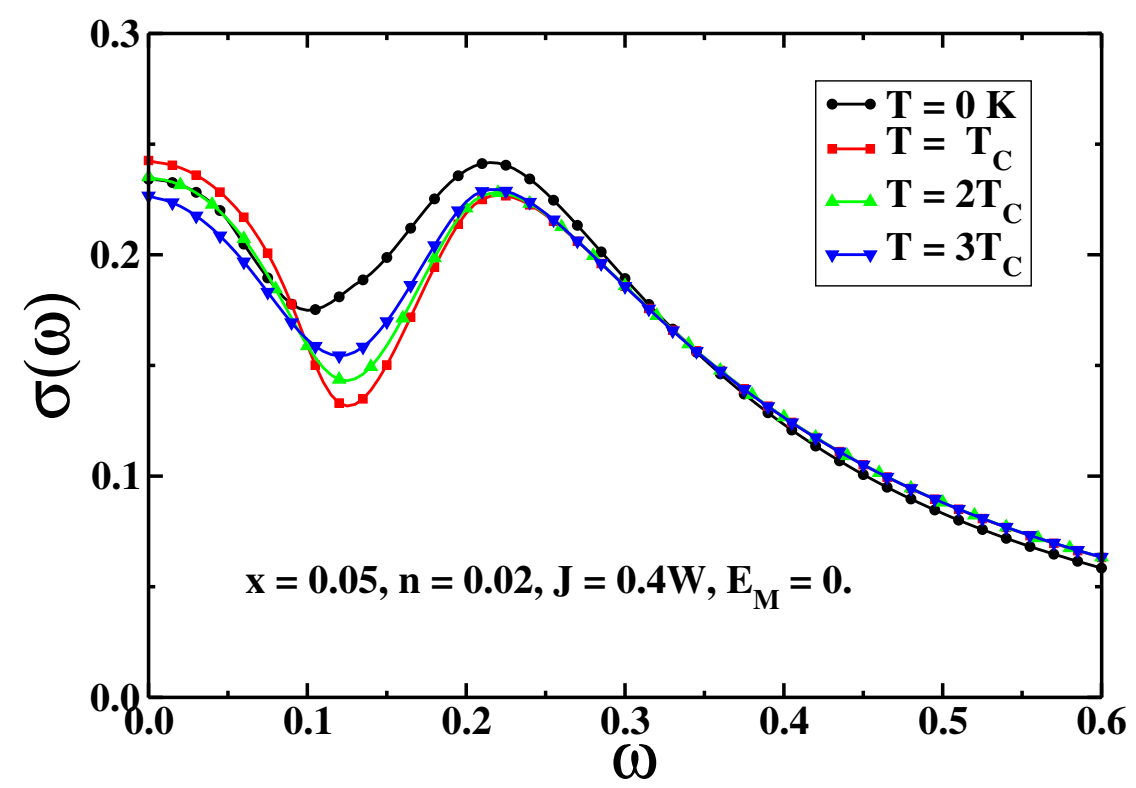

Figure 3: Temperature dependence of the conductivity

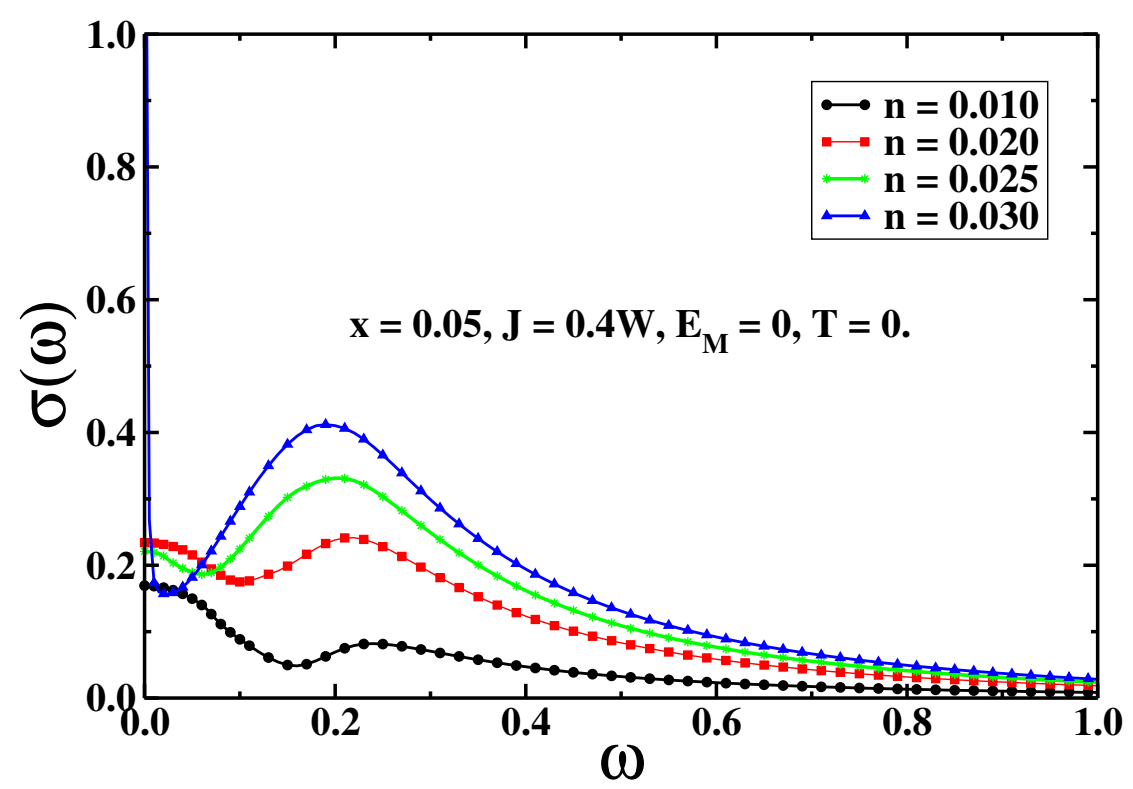

Figure 4: Density dependence of the conductivity 


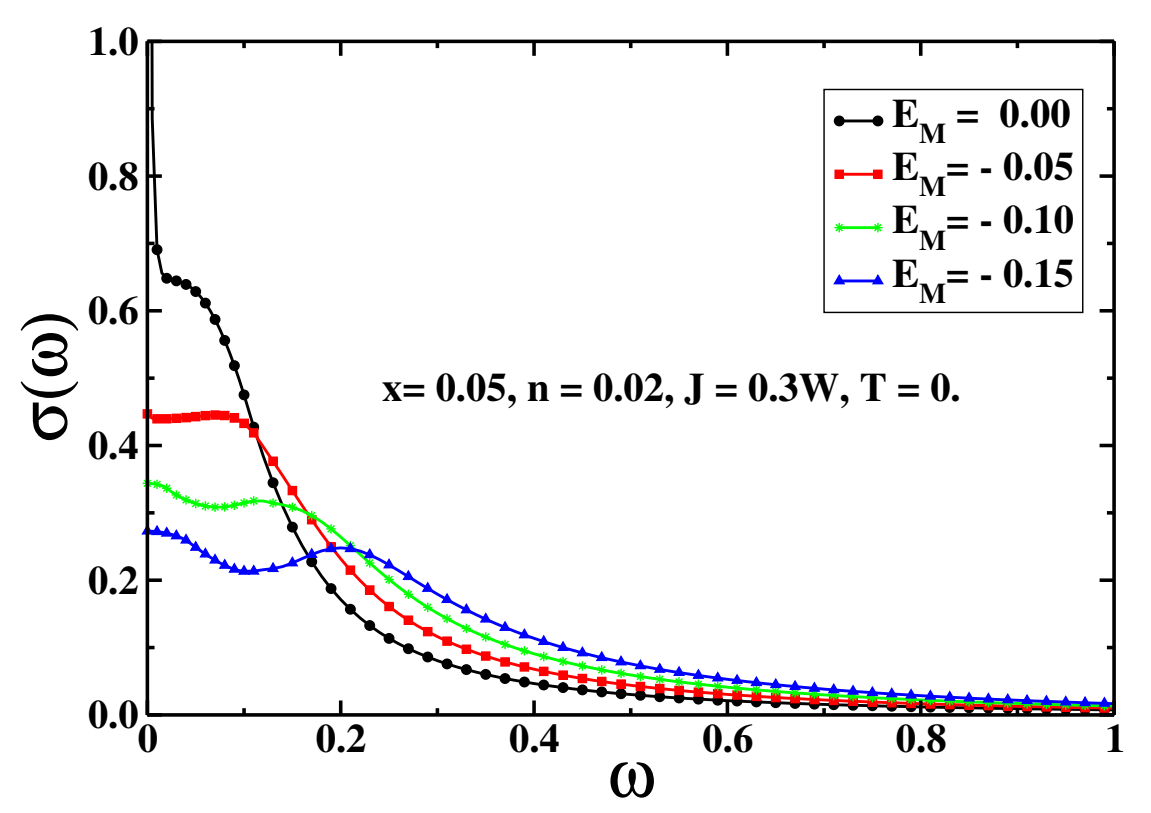

Figure 5: nonmagnetic potential dependence of the conductivity

use the parameter $J=0.4 W, E_{M}=0$ and $n=0.02$. Unlike the results from classical impurity spin models $[11,12]$, as $T$ is increased the finite peak is unchanged in energy (in good agreement with experiments), while it moves down in intensity for $T \leq T_{c}$ and nearly remains constant for $T_{c} \leq T \leq 3 T_{c}$ (somewhat different from experiments). This disrepancy may be caused by neglecting the complicated band structure in the model as well as nonlocal effecs in CPA calculation. The density dependence of the optical conductivity for $x=0.05, T=0, J=0.4 W, E_{M}=0$ is shown in Fig. 4 and it is as follows: as the density increases the conductivity increases and the finite-frequency peak moves down in energy. The shift of finite-frequency peak to the left is caused by the decrease in separation between the chemical potential and the edge of the main band (as $n$ is increased). Fig. 5 displays the nonmagnetic potential dependence of the conductivity. Comparing with the curves in Fig. 2 it is clear that $E_{M}$ increases the effective value of $J$. Our results agree with $[5,20]$ and confirm that the nonmagnetic potential simply renormalizes $J$.

To summarize, in this paper using CPA and treating the impurity spins fully quantum mechanically within the Weiss molecular theory, we have investigated the optical conductivity in diluted magnetic semiconductors (III,Mn)V for a wide range of model parameters. We have shown that the optical conductivity strongly depends on the temperature and the system parameters, i. e., exchange coupling, carrier density, nonmagnetic potential. By taking into account the quantum spin effect, our results are in reasonable agreement with the ones obtained by $\mathrm{MC}$ simulation, and support the view that a broad peak at around $0.2 \mathrm{eV}$ experimentally observed in the optical conductivity of $\mathrm{Ga}_{1-x} \mathrm{Mn}_{x} \mathrm{As}$ 
for $x=0.052$ is due to transition from the impurity band to the main band. Despite of some shortcomigs of the model and formalism (the neglecting of the band structure and nonlocal effects) we believe that such a description can still catch the essential physics of DMS.

\section{ACKNOWLEDGMENTS}

This work was done during a visit of A. T. Hoang to the Max Planck Institute for the Physics of Complex Systems, Dresden. He would like to thank Prof. P. Fulde and Dr. M. S. Laad for uselful discussions. Thanks are to the MPI PKS for hospitality and support. This work is partly supported by the National Program for Basic Research on Natural Science.

\section{References}

[1] H. Ohno, Science 281 (1998) 951.

[2] G. Tang and W. Nolting, Phys. Rev. B75 (2007) 024426.

[3] T. Jungwirth, J. Sinova, J. Masek, J. Kucera, A.H. MacDonald, Rev. Mod. Phys. 78 (2006) 809.

[4] M. Takahashi and K. Kubo, Phys. Rev. B66 (2002) 153202.

[5] M. Takahashi and K. Kubo, J. Phys. Soc. Jpn. 72 (2003) 2866.

[6] G. Bouzerar, J. Kudrnovsky, and P. Bruno, Phys. Rev. B68 (2003) 205311.

[7] S. Feng and M. Mochena, J. Phys.: Condens. Matter 18 (2006) 1441.

[8] S. Feng and M. Mochena, Physica B390 (2007) 79.

[9] A. Chattopadhyay, S. Das Sarma, and A. J. Millis, Phys. Rev. Lett. 87 (2001) 227202.

[10] G. Alvares and E. Dagotto, Phys. Rev. 68 (2003) 045202.

[11] A. T. Hoang and D.A. Le, Mod. Phys. Lett. B21 (2007) 69.

[12] E.H. Hwang, A.I. Millis, and S. Das Sarma, Phys. Rev. B65 (2002) 233206.

[13] M. Takahashi and K. Mitsui, Phys. Rev. B54 (1996) 11298.

[14] B. Velicky, Phys. Rev. 189 (1969) 614.

[15] R. Auslender and E. Kogan, Eur. Phys. J. B19 (2001) 525.

[16] A. J.H. Park, S.K. Kwon, B.I. Min, Physica B. 281/282 (2001) 703.

[17] E.J. Singley, R. Kawakami, D.D. Awschalom, D.N. Basov, Phys. Rev. Lett. 89 (2002) 097203.

[18] K. Hirakawa, S. Katsumoto, T. Hayashi, Y. Hatshimoto, and Y. Iye, Phys. Rev. B65 (2002) 193312.

[19] F. Popescu, Y. Yildirim, G. Alvares, A. Moreo, and E. Dagotto, Phys. Rev. B73 (2006) 075206.

[20] F. Popescu, C. Sen, E. Dagotto, and A. Moreo, Cond-mat/0705.039v1. 